\title{
Ethnologies
}

\section{Chaire de recherche du Canada sur les patrimoines ethnologiques des francophones d'Amérique (xvie-xxie siècles)}

\section{Laurier Turgeon}

Volume 26, numéro 2, 2004

Québec - Ethnologie du proche

Québec - Ethnology At Home

URI : https://id.erudit.org/iderudit/013752ar

DOI : https://doi.org/10.7202/013752ar

Aller au sommaire du numéro

Éditeur(s)

Association Canadienne d'Ethnologie et de Folklore

ISSN

1481-5974 (imprimé)

1708-0401 (numérique)

Découvrir la revue

Citer cette note

Turgeon, L. (2004). Chaire de recherche du Canada sur les patrimoines

ethnologiques des francophones d'Amérique (xvie-xxie siècles). Ethnologies,

26(2), 291-300. https://doi.org/10.7202/013752ar 


\title{
Chaire de Recherche du CANAdA SUR Les PATRIMOINES ETHNOLOGIQUES DES FRANCOPHONES D’AMÉRIQUE (XVI'-XXIe SIÈCLES) ${ }^{1}$
}

\author{
Laurier Turgeon \\ Titulaire de la Chaire de recherche du Canada en patrimoine ethnologique \\ Université Laval, Québec
}

\section{Les objectifs de la Chaire}

La Chaire sur les patrimoines ethnologiques des francophones d'Amérique est une chaire de niveau I financée pour une période de sept ans (2003-2010) par le Conseil de recherche en sciences humaines du Canada. Elle poursuit quatre objectifs.

Le premier, d'ordre théorique, vise à renouveler le concept de patrimoine ethnologique pour l'adapter aux réalités et aux préoccupations du $\mathrm{XXI}^{\mathrm{e}}$ siècle. Nous voulons remettre en question les notions d'authenticité, de tradition et d'identités originaires, généralement rattachées au patrimoine ethnologique, pour reconceptualiser ce dernier en mettant l'accent sur le mouvement, les mutations et les mélanges. Loin d'être figé, le patrimoine ethnologique est continuellement reconstruit par les contacts, les échanges et les interactions entre individus et groupes différents. En plus de faire ressortir le caractère dynamique du patrimoine, cette approche neuve permettra de contribuer aux études culturelles sur la mondialisation, le transnationalisme et le métissage, qui représentent aujourd'hui un courant théorique majeur en sciences humaines.

Le deuxième objectif, méthodologique celui-ci, veut prendre comme champ d'observation le patrimoine des francophones

1. www.patrimoine.chaire.ulaval.ca 
d'Amérique. Le choix de ce terrain élargi permet d'étudier les francophones de vieille souche, les nouveaux francophones issus de l'immigration et aussi les francophones d'adoption, c'est-à-dire ceux provenant d'une autre culture qui choisissent le français comme langue première. Le patrimoine des francophones d'Amérique offre un terrain d'une grande originalité pouvant éclairer la réflexion actuelle sur le transnationalisme et le métissage culturel en raison de ses nombreux contacts avec le patrimoine d'autres groupes (Amérindiens, Anglais, Irlandais, etc.), des mélanges culturels engendrés et de son caractère diasporique. Par notre démarche résolument interdisciplinaire, nous serons à même, d'abord, de déconstruire l'histoire du chassé-croisé des emprunts culturels des francophones d'Amérique (XVI $-\mathrm{XXI}^{\mathrm{e}}$ siècle), à partir d'un corpus élargi (sources manuscrites, imprimées, matérielles, orales et iconographiques) et, ensuite, de construire une histoire relationnelle de ce patrimoine, centrée sur les métissages et les syncrétismes.

Le troisième objectif, d'ordre pédagogique, est destiné à fournir aux chercheurs, aux postdoctorants et aux étudiants : 1) un encadrement interdisciplinaire de haut niveau, 2) un forum qui favorise les débats et l'élargissement de nos perspectives de recherche, 3) un lieu propice à la diffusion de travaux (colloques, conférences, ateliers, publications, etc.), 4) des bourses de deuxième et de troisième cycles ainsi que des bourses postdoctorales.

Enfin, la Chaire veut contribuer à faire de l'Université Laval un centre international d'études sur le patrimoine. Elle aidera à promouvoir et à fédérer, en lien étroit avec l'Institut du patrimoine culturel (IPAC), les recherches en matière de patrimoine des centres, des autres chaires et des groupes de recherche de la Faculté des lettres, puis à fonder un laboratoire d'enquêtes et d'entrevues multimédia, grâce à une subvention d'infrastructure de la FCI.

\section{Problématique de la recherche}

Le patrimoine ethnologique évoque l'idée de catégories ethnoculturelles étanches et d'identités originaires enracinées dans des lieux et des temps immuables. Il présente souvent la transmission et la conservation comme une lutte contre la dégradation, la disparition ou la destruction. Il exprime un désir de récupération et de restauration des restes, mais il traite peu de la création. Il observe ce qui disparait, 
encore trop rarement ce qui apparaît (Chiva 1990 : 236). Pendant longtemps, le patrimoine a suscité une pratique de gestion des biens familiaux — des «biens du père » selon l'étymologie du mot. Il a été mis au service de l'État, qui l'a confondu avec la patrie et qui a agi avec l'idée que la transmission des «biens de la nation " assure la continuité et la stabilité de la communauté nationale (Lowenthal 1985, 1996 ; Poulot 1998, 2001). Dans le cadre actuel de la mondialisation, une politique du patrimoine se veut un moyen efficace pour protéger les sites classés contre les effets des conflits armés ou d'un tourisme excessif, et aussi pour préserver les biens patrimoniaux locaux de la menace d'une homogénéisation des cultures (UNESCO 2002). Si la «patrimonialisation » des biens collectifs reflète et suscite des «passions identitaires ", un attachement émotionnel au monde, à la nation ou à la famille (Le Goff 1998), elle tend à les présenter comme des choses « déjà-là », autodéterminées, de manière linéaire et téléologique. En exaltant la patrie, les préoccupations patrimoniales ont essentiellement porté sur la transmission de soi à soi, beaucoup moins de soi à l'autre ou de l'autre à soi. La patrimonialisation sélectionne les biens à transmettre et leur accorde un statut, mais elle explique peu comment le patrimoine se construit et se transforme. Les chercheurs situent généralement le patrimoine dans des contextes régionaux, nationaux et internationaux, mais à peu près jamais dans les contextes coloniaux et postcoloniaux. Le patrimoine tend à être présenté comme un héritage du passé qui se produit au centre des nations ou des empires, plus rarement dans leurs marges.

Nous voulons faire ressortir le caractère dynamique du patrimoine ethnologique, en mettant l'accent sur le mouvement, les mutations et les mélanges. Le patrimoine ethnologique, comme le patrimoine en général, est continuellement reconstruit par les contacts, les interactions, et les échanges entre individus et groupes différents (Amselle 1990, 2001 ; Clifford 1997 ; Jeudy 1990). La transmission implique la médiation et l'appropriation ; la conservation entraîne la transformation (Lamizet 1999 ; Poulot 2001). Les objets échangés, les personnes déplacées ou les lieux occupés subissent des recontextualisations culturelles : ils prennent d'autres formes, ils acquièrent de nouveaux usages et ils changent de sens (Meyers 2001). Transformer ou modifier leur usage devient une manière de marquer une appropriation. Les objets échangés transforment ceux-là mêmes qui les manipulent. Aussi, la transmission et la prise de possession d'objets nouveaux provoquent 
non seulement des reconfigurations culturelles, mais également le reclassement et la redéfinition d'individus et de groupes dans la société. Le patrimoine découle d'un processus de métissage culturel qui naît d'une dynamique relationnelle entre le soi et l'autre, d'une dialectique de la différence qui permet aux deux entités de se constituer l'une et l'autre (Ricœur 1990). Plus encore, les pratiques patrimoniales produisent l'autre pour le ramener vers soi, pour mieux se l'approprier. Le patrimoine définit donc un champ interactif qui permet au sujet de se construire. Plus qu'un lieu ou une chose, le patrimoine est un mouvement, un déplacement de l'autre vers soi, l'expression d'une force, donc le vecteur d'enjeux sociaux et idéologiques. Sensible aux mélanges de l'héritage culturel du Québec et à la diversité des cultures qui ont occupé ce territoire, le groupe-conseil sur la Politique du patrimoine culturel du Québec, présidé par Roland Arpin, a proposé le développement du concept de métissage en matière de patrimoine du Québec et de l'Amérique française (Arpin 2000 : 11-12).

\section{Axes et méthodes de recherche}

Ce programme de recherche s'inscrit dans le prolongement d'une réflexion entamée, il y a une douzaine d'années, dans le cadre d'une étude sur les échanges entre Français et Amérindiens en NouvelleFrance. À partir d'un corpus élargi (sources manuscrites, imprimées, matérielles et iconographiques), nous nous sommes efforcé de construire une histoire relationnelle de ces deux groupes, centrée sur les contacts et les échanges plutôt que sur les groupes ethnoculturels eux-mêmes. Plus récemment, nous avons étendu le champ chronologique à l'époque contemporaine pour voir comment les pratiques du métissage de l'époque coloniale se brisaient ou se poursuivaient dans la société postcoloniale. C'est cette perspective comparée très éclairante entre sociétés coloniales et postcoloniales que nous voulons explorer avec plus de détermination pour savoir, entre autres, comment le patrimoine ethnologique de l'époque coloniale est réinterprété dans le contexte postcolonial. La présente Chaire de recherche nous permettra d'élaborer et d'étendre notre modèle interprétatif à d'autres dimensions du métissage, notamment dans le champ du politique, d'agrandir notre base documentaire (surtout par l'ajout de sources orales) et d'étendre le cadre chronologique aux $\mathrm{XX}^{\mathrm{e}}$ et $\mathrm{XXI}^{\mathrm{e}}$ siècles. 
Pour faciliter la mise en œuvre de notre programme de recherche, nous l'avons orienté selon trois axes. Le premier vise à théoriser le patrimoine ethnologique en regard du métissage. Nous voulons mettre à contribution et développer la réflexion théorique sur le métissage culturel (Amselle 1990, 2001; Gruzinski 1999 ; Laplantine et Nouss 1997, 2001), pour explorer les manières dont l'hybridité intervient dans la construction et la transmission du patrimoine. Tout métissage s'effectue dans un espace de contact qui n'est pas un simple lieu de croisements et de rencontres heureuses, mais un champ de tension où des stratégies et des forces s'affrontent autour d'une frontière physique ou symbolique. C'est donc un espace relationnel qui met en œuvre des négociations, des stratégies d'appropriation et des postures de résistance. Lieux de tensions, les espaces de contact sont aussi, comme le rappelle Homi Bhabha, des espaces de création, des « entre-lieux » de la culture, où se réalisent de nouveaux consensus, de nouvelles pratiques culturelles et de nouvelles identités (Bhabha 1994 : 1-2). Le métissage n'est pas envisagé ici comme une simple transition entre l'avant et l'après-contact, ni comme une étape, de durée limitée, dans un processus d'assimilation qui conduit à la disparition de l'une des deux cultures. À l'instar d'Amselle, nous le considérons comme un processus continuel d'interaction entre deux ou plusieurs cultures qui se transforment mutuellement. Dans cette perspective, les cultures ne sont pas des entités stables, mais des systèmes déjà constitués de manière relationnelle. Loin de situer le métissage sur les marges et dans une temporalité restreinte, nous voulons l'inscrire au centre de tout processus culturel et comme fondement du patrimoine.

Le deuxième axe porte sur les processus historiques du métissage des patrimoines ethnologiques en prenant comme champ d'observation les francophones d'Amérique. Plutôt que de nous limiter aux seuls francophones de souche, nous voulons embrasser tous ces groupes qui ont été en contact avec les francophones et qui, dans certains cas, se sont intégrés à la francophonie. Comme les processus de métissages s'étirent souvent sur de longues périodes, il faut prendre en compte le temps pour les observer. L'histoire offre un vaste terrain ethnologique qui permet de suivre les déplacements et les recontextualisations culturelles des biens patrimoniaux dans la longue durée. C'est l'étude du mouvement de ces biens dans le temps qui éclaire les multiples usages auxquels ils ont été destinés. La démarche que nous voulons 
mettre en œuvre vise à isoler les objets ou les sites patrimoniaux pour mieux reconstituer leur genèse puis leur parcours, dans le temps et l'espace, d'un groupe culturel à un autre. Le patrimoine est polyréférentiel, car approprié par divers groupes et mobilisé par différents projets politiques et idéologiques. Ainsi, il n'y a pas un patrimoine, mais des patrimoines ethnologiques. On remontera souvent à l'époque de la colonisation européenne de l'Amérique, en raison de l'intensité des contacts et du rôle important que joue encore aujourd'hui le patrimoine ethnographique de cette période. Les archives et les musées de l'Amérique française renferment des collections entières d'archives et d'objets (amérindiens, français, anglais, irlandais) de l'époque coloniale, qui sont régulièrement exposés au grand public. Il s'agira de suivre le mouvement des objets et des personnes pour reconstituer les chaînes de transmission et de transformation qui donnent aujourd'hui sens aux biens patrimoniaux. Le lien constant qui est fait avec le présent permet aussi de repérer les nouveaux sites de contact et les nouveaux lieux de production des patrimoines dans nos sociétés postcoloniales. Le passé sert à informer le présent et le présent permet de mieux interroger le passé. Plutôt que de construire un méta-texte historique qui prétend englober tout le passé, on procédera par études de cas ou de lieux, chacun de ces lieux étant interrogé à partir de plusieurs perspectives disciplinaires. Isoler des biens patrimoniaux permet de cerner le fonctionnement concret de leur métissage dans le temps. Par exemple, nous voulons développer et raffiner une méthode que nous appelons « récits d'objets ", qui consiste à reconstituer la "généalogie » des objets patrimoniaux à l'aide de documents d'archives et des récits oraux de leurs propriétaires. En plus de fournir un puissant appareil d'information et de croisements de données, cette approche permet d'intégrer l'étude de la culture matérielle, la recherche en archives et le témoignage oral, opérations par ailleurs trop souvent cloisonnées à l'intérieur de pratiques disciplinaires. Une priorité sera accordée, au moins en début de projet, à l'étude du patrimoine des communautés religieuses en raison de la menace qui pèse sur lui à cause du vieillissement des membres de nombreux ordres religieux.

Le troisième axe est destiné à appréhender les enjeux que représentent les métissages du patrimoine ethnologique dans le contexte actuel de la mondialisation. Nous voulons ici cerner et mieux comprendre les aspects idéologiques du métissage. Une esthétique de l'hétérogène tend, aujourd'hui, à patrimonialiser le métissage et à l'ériger 
en une idéologie au service de la mondialisation. Si, à l'origine, la notion de métissage a été utilisée pour lutter contre l'ethnicité et le fondamentalisme, elle est maintenant valorisée à tel point qu'elle est en passe de devenir une esthétique universelle, acceptée et partagée par tous, voire imposée aux autres. L'hybridité est célébrée par les critiques d'art et elle devient un bien de consommation. La diversité culturelle se vend bien. Le métissage, comme le multiculturalisme d'ailleurs, peut donner bonne conscience aux élites en leur procurant un sentiment de rectitude morale et d'engagement social, alors que, souvent, il ne fait que consolider les hégémonies en place. Pourtant, le métissage ne produit pas toujours du beau et il n'est pas forcément libérateur, même si l'articulation d'un patrimoine authentique et autonome peut devenir, pour les minorités dominées, l'affirmation d'une résistance plutôt qu'une simple expression d'ethnocentrisme et d'autochtonie. Il faut donc bien se garder de fabriquer des outils rhétoriques qui construiraient de nouveaux essentialismes. L'étude des contextes est essentielle car ce qui importe sur le plan politique dépend de l'entité qui instaure le métissage ou l'authenticité, et de son pouvoir à maintenir une hégémonie.

\section{Le Laboratoire d'enquête ethnologique et de multimédia}

Le Laboratoire d'enquête ethnologique et de multimédia est destiné à offrir à la Chaire de recherche du Canada sur les patrimoines ethnologiques des francophones d'Amérique $\left(\mathrm{XVI}^{\mathrm{e}}-\mathrm{XXI}^{\mathrm{e}}\right.$ siècle) un lieu centralisé pour la cueillette, le traitement, la conservation et la diffusion des données recueillies et pour la formation des étudiants et des chercheurs. L'infrastructure fournit un espace doté d'équipements modernes (multimédia) ainsi qu'une expertise pour l'apprentissage (formation) des technologies de pointe liées aux méthodologies de réalisation des enquêtes, de leur traitement, de leur conservation et de leur diffusion. Les ressources du laboratoire permettent d'effectuer des enquêtes sous plusieurs formes, de divers types et sur des supports variés : audio, visuel, analogique, numérique, grâce aux nouvelles technologies. Il regroupe l'ensemble des technologies nécessaires pour la saisie des entrevues et leur traitement.

De façon plus précise, l'infrastructure comprend une salle munie de quinze postes de travail et de deux projecteurs LCD (l'un fixe et l'autre portatif), pour traiter et visualiser les données recueillies sur le terrain. 
Chaque poste est équipé d'un ordinateur, d'un lecteur minidisc, d'un micro et d'un appareil photo numérique (partagé) ou d'une caméra numérique (partagée). L'usage de micro-ordinateurs portatifs, équipés de micros, de caméras ou d'appareils photos, donne l'occasion à l'enquêteur d'enregistrer textes, son et images pendant une même sortie et de commencer le traitement des données sur le terrain. Ces équipements offrent donc à l'enquêteur, en plus de l'autonomie, une plus grande mobilité, des enregistrements numériques de grande qualité, une efficacité accrue dans la cueillette et le traitement des données et une meilleure conservation de l'information.

Un tel laboratoire d'ethnologie spécialisé en enquête et doté d'équipements multimédias représente une capacité de recherche nationale et internationale unique puisque, à notre connaissance, il n'en existe pas ailleurs, ni au Canada, ni en Amérique du Nord, ni en Europe. L'expertise développée à l'Université Laval en matière de traitement et de conservation des archives sonores, notamment par le service des Archives de folklore et d'ethnologie (AFEUL) depuis 1944 et par le programme d'enseignement en ethnologie (formation à l'enquête) depuis 1971, permet de développer et de positionner notre laboratoire spécialisé sur le plan national et international. Forte de ce savoir-faire, l'Université Laval et ses ressources actuelles s'imposent naturellement comme le lieu d'accueil privilégié pour mettre en place cette nouvelle infrastructure. Le laboratoire est situé à proximité des Archives de folklore et d'ethnologie (sixième étage du Pavillon Bonenfant) pour avoir plus facilement accès à leurs fonds et pour bénéficier de leurs services spécialisés. 


\section{Références}

Amselle, Jean-Loup, 1990, Logiques métisses : anthropologie de l'identité en Afrique et ailleurs. Paris, Payot.

—_, 2001, Branchements. Anthropologie de l'universalité des cultures. Paris, Flammarion.

Appadurai, Arjun, 1997, Modernity at Large : Cultural Dimensions of Globalization. Minneapolis, University of Minnesota Press.

Arpin, Roland (sous la présidence de), 2000, Notre patrimoine, un présent du passé. Québec, Communication Science-Impact.

Bhabha, Homi K., 1994, The Location of Culture. London and New York, Routledge.

Cardin, Martine, 2002, «Présentation de l'Institut sur le patrimoine culturel ». Dans Marie-Claude Rocher (dir.), La pertinence sociale du patrimoine dans la Cité contemporaine, Québec, Communication Science-Impact : 61-73.

Chiva, Isac, 1990, « Le patrimoine ethnologique : l'exemple de la France ». Encyclopédia Universalis tome $24: 236$.

Clifford, James, 1997, Routes, Travel and Translation in the Late Twentieth Century. Cambridge, Harvard University Press.

Desdouits, Anne-Marie et Laurier Turgeon, 1997, «Introduction». Dans Anne-Marie Desdouits et Laurier Turgeon (dir.), Ethnologies francophones de l'Amérique et d'ailleurs, Québec, Les Presses de l'Université Laval : vii-xxviii.

Gruzinski, Serge, 1999, La pensée métisse. Paris, Fayard.

Jeudy, Henri-Pierre (dir.), 1990, Patrimoines en folie. Paris, Maison des Sciences de l'Homme.

Lamizet, Bernard, 1999, La médiation culturelle. Paris, L'Harmattan.

Laplantine, François et Alexis Nouss, 1997, Le métissage. Paris, Flammarion.

—_, 2001, Métissage, d'Arcimboldo à Zombi. Paris, Pauvert.

Le Goff, Jacques, 1998, «Introduction des Entretiens du Patrimoine ».

Dans Jacques Le Goff (dir.), Patrimoines et passions identitaires, Paris, Fayard et Éditions du patrimoine.

Lowenthal, David, 1985, The Past is a Foreign Country. Cambridge University Press.

- 1998, Possessed by the Past. New York, Free Press.

Meyers, Fred R. (dir.), 2001, The Empire of Things: Regimes of Value and Material Culture. Santa Fe, School of American Research Press.

Ministère de la Culture (France), (n.d.), Mission du Patrimoine ethnologique de la France. http//www.culture.fr/mpe. 
Poulot, Dominique, 2001, Patrimoine et musées : L'institution de la culture. Paris, Hachette.

—_ 1998, «Introduction ». Dans Dominique Poulot (dir.), Patrimoine et modernité. Paris, L'Harmattan.

Ricœur, Paul, 1990, Soi-même comme un autre. Paris, Seuil.

Unesco, 2002, Nouvelles notions du patrimoine: itinéraires culturels. (En ligne)http //: mirror-us.unesco.org 